\title{
Non-linear diffusion of Cosmic Rays escaping from Supernovae Remnants in the cold partially neutral molecular and atomic phases
}

\author{
Loann Brahimi* \\ LUPM (Montpellier, France) \\ E-mail: loann.brahimieumontpellier.fr
}

\section{Alexandre Marcowith}

LUPM (Montpellier, France)

E-mail: alexandre.marcowitheumontpellier.fr

\section{Vladimir Ptuskin}

Pushkov Institute of Terrestrial Magnetism, Ionosphere and Radiowave Propagation (Troitsk, Moscow, Russia)

We study Cosmic Ray (CR) propagation in the weakly ionized environments of supernovae remnants (SNRs). Our work is based on a Cosmic Ray Cloud (CRC) model developed by Malkov et al. (2013) and Nava et al. (2016). We solve two transport equations simultaneously: one over the CRs pressure and one for the Alfvén waves energy density. CRs are initially confined by the SNRs shock. While escaping from their accelerator, CRs start to trigger the streaming instability and produce slab-type resonant Alfvén modes. The self-generated turbulence is damped by ionneutral collision and by non-correlated interaction with Alfvén modes generated at large scale. We show that CRs leaking in cold dense phases as Cold Neutral Medium (CNM) and Diffuse molecular Medium (DiM) can still be confined over distances of a few tens of parsecs from the CRC center for a few kyrs. At $10 \mathrm{TeV}$ CR diffusion can be suppressed by two or three orders of magnitude. This effect results from a reduced ion-neutral collision damping in the low frequency regime. We calculate the grammage of $\mathrm{CR}$ in these environements. We find both in single or multi-phase set ups that at $10 \mathrm{GeV} \mathrm{CNM}$ and DiM media can produce grammage in the range $10-20 \mathrm{~g} / \mathrm{cm}^{2}$ in the CNM and DiM phases. At $10 \mathrm{TeV}$ because of non-linear propagation the grammage increases to values in the range $0.5-20 \mathrm{~g} / \mathrm{cm}^{2}$ in these two phases. We also discuss preliminary calculations in inhomogeneous ISM combining two or three different phases where we obtain the same trends. Finally, we discuss some improvements of the CRs diffusion code : Increase of the dimensionnality of the problem, inclusion of perpendicular diffusion effects, interstellar cloud ionization and gamma-ray emission.

36th International Cosmic Ray Conference -ICRC2019-

July 24th - August 1st, 2019

Madison, WI, U.S.A.

${ }^{*}$ Speaker. 


\begin{tabular}{cccc} 
Phase & WNM & CNM & DiM \\
\hline$T[\mathrm{~K}]$ & $8 \times 10^{3}$ & 50 & 50 \\
$n_{\mathrm{T}}\left[\mathrm{cm}^{-3}\right]$ & 0.35 & 30 & 300 \\
$X$ & 0.02 & $8 \times 10^{-4}$ & $10^{-4}$
\end{tabular}

Table 1: Fiducial physical quantities for the WNM, CNM and DiM phases adopted in this work. $T$ is the gas temperature, $n_{\mathrm{T}}=n_{\mathrm{n}}+n_{\mathrm{i}}$ is the total gas density, $X$ is the gas ionization fraction.

\section{The model}

In this work, we use an analytical model developped by Malkov et al. (2013) which proposes that the CRs are emitted via diffusive shock acceleration (DSA) and propagate mainly along the galactic magnetic field lines by generating resonant linearly polarized transversal magnetic pertubations moving along the mean field lines. CRs, by this way, generate the turbulence they scatter off. We call this effet the self generated turbulence. This induces a reduction of the CRs streaming by confining them close to their accelerator. In the meantime, the generated waves interact with the background plasma and with the turbulence generated at larger scales $(>50 \mathrm{pc})$ via non-correlated interferences (Lazarian 2016). In order to numerically probe this effect, we need to combine different models. A model of CRs leakage from by the source, a model of ISM, a model of interaction between CRs and magnetic turbulence. We describe all these below.

We consider the problem in 1D. The cylindric geometry is the best approximation at first order as proposed by Malkov et al. (2013). We therefore need to discuss the limits of this approximation. The characteristics scales of the magnetic field lines (in space $L_{c}$ and time $T_{c}$ ) need to be larger than the characteristics scales of the simulations $\left(L_{c}>2000 \mathrm{pc}\right.$ and $\left.T_{c}>1 \mathrm{Myr}\right)$. We only consider parallel CR diffusion. We do not consider non-parallel modes that can modify the CRs diffusion coefficient especially in the energy range where they do not produce any turbulence (see section $1.1)$.

\subsection{The ISM model}

The interstellar medium is modeled using three different phases of partially ionized medium (see table 1) : the Warm Neutral Medium (WNM), the Cold Neutral Medium (CNM) and the Diffuse Molecular cloud (DiM) (see table 1).

In order to quantify the interaction of the magnetic waves with the plasma, we based our approach on the resolution of an MHD bi-fluid plasma dispersion relation (see Xu et al. 2016, Soler et al. 2013, Lazarian et al. 2004). The magnetic perturbations are supported by the ion fluid and they are damped by transfering energy to the neutral fluid via an ion-neutral collision rate given by $v_{\text {in }}=2 n_{n}\left(8.4 \times 10^{-9} \mathrm{~cm}^{-3} \mathrm{~s}^{-1}\right)\left(\frac{T}{10^{4} \mathrm{~K}}\right)^{0.4}$ for plasmas temperature $T>50 \mathrm{~K}$ and $v_{\text {in }}=n_{n}\left(2.1 \times 10^{-9} \mathrm{~cm}^{-3} \mathrm{~s}^{-1}\right)$ for colder plasmas (see Jean et al. 2009, Nava et al. 2016). Then, from the dispersion relation of the Alfvén waves we can obtain an ion-neutral collision damping term $\Gamma_{\mathrm{IN}}(k)$ depending on the CRs Larmor radius $r_{l}$ resonant scale $k=r_{l}^{-1}\left[\mathrm{~cm}^{-1}\right]$. We obtain two damping regimes corresponding to the scales where ions and neutrals have decorrelated interactions 
and the energy transfert rate is important (at large scales, $\Gamma_{\mathrm{IN}}=-v_{\text {in }} / 2$ ) and to scale where ions and neutrals have correlated interactions and the energy tranfert rate decreases as $E^{-2}$ (at small scales). Between these scales, the Alfvén waves dispersion relation does not have any real solution so waves do not propagate and no turbulence can be generated by CRs. In the meantime, the magnetic perturbations undergo decorelated interferences from the turbulence generated at large scale produces to a complementary damping of the waves (Lazarian 2016). The damping term strongly depends on the Alvén waves velocity $\Gamma_{\mathrm{L} 16}(k) \approx V_{\mathrm{A}}(k) / k^{-0.5} L^{0.5}$ if $k^{-1}<L . L=50 \mathrm{pc}$ is a turbulence injection scale and $V_{\mathrm{A}}(k)$ is the Alfvén waves velocity derived from the real solution of the plasma relation dispersion. This term starts to dominate the global waves damping term only at high energies $(>10 \mathrm{TeV})$.

\subsection{Interactions between CRs and magnetic perturbations}

As proposed by Nava et al. (2016), we treat our CRs flux according to a transport equation over the pressure $P_{\mathrm{CR}}$. Waves magnetic energy density perturbations $I(k)=\left(\delta B / B_{0}\right)^{2}$ are solved using a second kinetic equation. We only consider resonnant generated waves $k=r_{\mathrm{L}}^{-1}$ where $k$ corresponds to the spatial wave number and $r_{\mathrm{L}}=\gamma m c / q B_{0}$ is the CRs Larmor radius. We describe the transport of CRs and waves along magnetic field lines by solving two coupled equations given by

$$
\frac{\partial P_{\mathrm{CR}}}{\partial t}+V_{\mathrm{A}} \frac{\partial P_{\mathrm{CR}}}{\partial z}=\frac{\partial}{\partial z}\left(D \frac{\partial P_{\mathrm{CR}}}{\partial z}\right)
$$

for the CR pressure $P_{\mathrm{CR}}$ and

$$
\frac{\partial I}{\partial t}+V_{\mathrm{A}} \frac{\partial I}{\partial z}=2\left(\Gamma_{\text {growth }}-\Gamma_{\mathrm{d}}\right) I+Q
$$

for the waves energy density $I$.

In equation (1.1), the right hand term corresponds to the CRs diffusion term where $D$ is the CRs diffusion coefficient. The term depends on the waves energy density $I$ by the relation $D=D_{\mathrm{B}} / I$ where $D_{\mathrm{B}}$ is the Bohm diffusion coefficient (see Bell 1978ab). We clearly see that a production of magnetic perturbations contribute to confine CRs via decreasing their diffusion coefficient. In equation (1.2), the term $\Gamma_{d}=\Gamma_{\mathrm{IN}}+\Gamma_{\mathrm{L} 16}$ corresponds to the damping term of waves via ion-neutral collisions $\left(\Gamma_{\mathrm{IN}}\right)$ and non-correlated interactions with the large scale turbulence $\left(\Gamma_{\mathrm{L} 16}\right)$. These damping terms are compensated by a source term $Q$ which corresponds to the injection of waves from the large scales. Finally, the term $\Gamma_{\text {growth }}$ corresponds to a wave production term by the streaming instability damping. This term is defined by the relation

$$
2 \Gamma_{\text {growth }} I=-\frac{V_{\mathrm{A}}}{W_{0}} \frac{\partial P_{\mathrm{CR}}}{\partial z}
$$

according to Skilling (1975). $W_{0}=B_{0}^{2} / 8 \pi$ is the magnetic energy density. $\Gamma_{\text {growth }}$ strongly depends on the CRs pressure gradient exerted over the plasma. The amount of generated waves and so the rate of CRs confinement is strongly dependent on the value of the initial CRs pressure gradient. Accordingly, the confinement effect is strongly dependent on the CRs injection model that we present below. 


\subsection{The CRs injection model}

We inject $10 \%$ of a SN mechanical energy into CRs with an energy power-law distribution index $\Gamma=2.2$ with $E_{\min }=2 \times 10^{-4} \mathrm{GeV}$ and $E_{\max }=2 \times 10^{4} \mathrm{GeV}$. CRs are initially confined in a region of size $a$. Malkov et al. (2013) define the CR Cloud (CRC) half-life time $t_{1 / 2}$ as the time it takes for the CR integrated pressure in the initial sphere to be divided by two. The CRC half time is defined by the relation

$$
\int_{-a}^{+a} P_{\mathrm{CR}}\left(z, t_{\frac{1}{2}}\right) \mathrm{d} z=2 a \frac{P_{\mathrm{CR}}^{0}}{2}
$$

where $P_{\mathrm{CR}}^{0}$ is the initial CR pressure in the cloud. To each CR energy corresponds a particular halflife time. In order to determine at which moment and at which SNR radius a given population of CR escapes, Nava et al. (2016) proposes to intersect the half-life time evolution function resulting from the equation (1.4) $t_{\frac{1}{2}}(a)$ with the solution $t\left(R_{\mathrm{SNR}}\right)$ describing the time evolution of a SNR radius (see more about SNRs shock evolution models in Cioffi et al. 1988, Truelove \& McKee 1999).

The results are shown in the figure (1.4). The right hand part of the figure shows that high energy CRs escape early while low energy CRs escape later when the magnetic turbulence inside the SNR shock becomes weaker. We also observe that in the CNM and the DiM, CRs escape earlier (between 10-100 times earlier) than in the WNM so the initial pressure gradient is higher and produces a stronger confinement.

\section{Numerical results}

Based on the CRs injection model described in the previous section, we solved the system of equations for waves and CRs. The results for $1 \mathrm{TeV}$ CRs and the resonant Alvén waves they produced are presented in the figure (2). The upper part of the figure shows the evolution in time (see the different color curves) and space of the CRs pressure around the remnant while the lower part shows the evolution of the CRs diffusion coefficent in time and space. Self generated turbulence produce a drop of the diffusion coefficient.

In the WNM, the CRC half-life time is about $1.5 \mathrm{kyr}$ and the escape radius is $6.4 \mathrm{pc}$. Consequently, the initial CRC pressure is about $2 \times 10^{-11} \mathrm{erg} / \mathrm{cm}^{3}$. At early times $(0.38 \mathrm{kyr})$ the $\mathrm{CR}$ diffusion coefficient drops by a factor of $2 \times 10^{3}$ from the background one. We still observe the self-confinement effect at $1.5 \mathrm{kyr}$. The non-linear effect relaxes after 6 kyrs. CRs follow a nonlinear diffusion process which rapidly relaxes after $4 \times t_{\frac{1}{2}}$.

In the CNM, the CRC half-life time is about $1.1 \mathrm{kyr}$ and the escape radius is $2.6 \mathrm{pc}$. The initial CRC pressure is higher than in the WNM $\left(P_{\mathrm{CR}}^{0}=3 \times 10^{-10} \mathrm{erg} / \mathrm{cm}^{3}\right)$. From $t_{\frac{1}{2}} / 4$ to $t_{\frac{1}{2}}$, the difference between numerical solutions and linear ones are important. The associated diffusion coeffient drops by a factor $5 \times 10^{3}$ from the background one. At late time $\left(4 \times t_{\frac{1}{2}}\right)$ the streaming instability starts to relax, but we clearly see the confinement effect through numerical and linear CRs pressure solutions. In the CNM the self-confinement effect is slightly stronger than in the WNM at $1 \mathrm{TeV}$ In the DiM, the CRC half-life time is about $3.0 \mathrm{kyrs}$ and the escape radius is $1.9 \mathrm{pc}$. Consequently the initial CRC pressure is higher than in the CNM $\left(P_{\mathrm{CR}}^{0}=10^{-9} \mathrm{erg} / \mathrm{cm}^{3}\right)$. As in the CNM, 

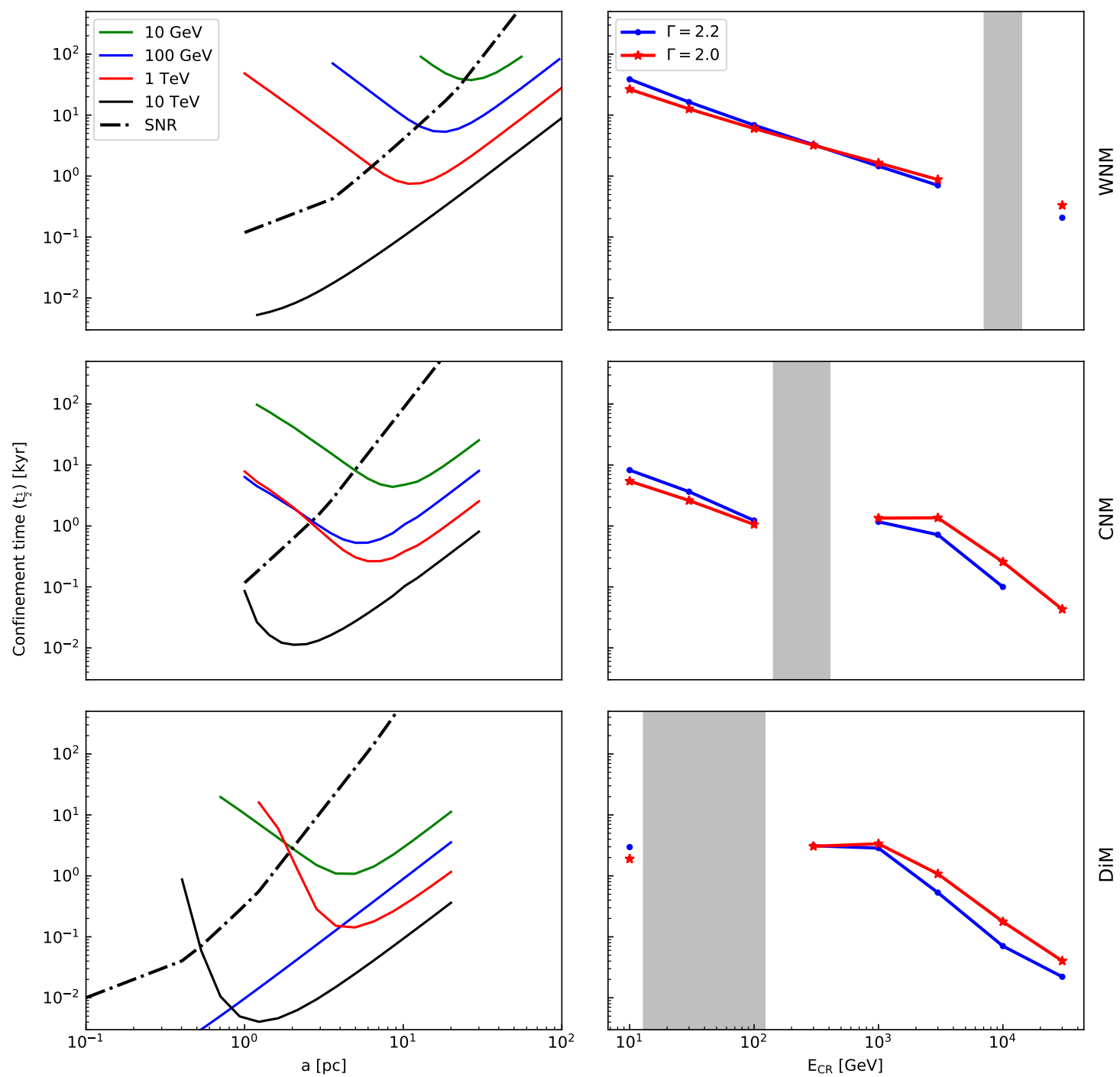

Figure 1: This figure shows the properties of the expansion of the CRC in the ISM. The left part of the figure represents the evolution of the half-life time of the CRC as a function of its initial radius $a$ in parsec units. Green, blue, red and black solid lines refer to particle energies at $10,10^{2}$, $10^{3}$ and $10^{4} \mathrm{GeV}$. The black dashed line refers to the time evolution of the radius of the SNR front shock in the considered phase: WNM for the top figure, CNM for the middle one and DiM for the bottom one. These plots have been obtained for $\Gamma=2.2$. The right part of the figure represents the evolution of the half-life time of the CRC derived from the left plots as a function of CR energies. Blue solid line refers to a CR energy spectral index of 2.2 while red dashed line refers to a spectral index of 2.0. The gray zone corresponds to the no-wave propagation zone, it does not lead to any $\left(a, t_{1 / 2}\right)$ solution. 
from $t_{\frac{1}{2}} / 4$ to $t_{\frac{1}{2}}$, the difference between numerical solutions and linear ones are important. The associated diffusion coeffient drops by a factor $8 \times 10^{3}$ from the background one. At late time $\left(4 \times t_{\frac{1}{2}}\right)$ the confinement effect is still strong, the diffusion coefficent increases less than a factor of ten. The difference between the linear solution and the numerical one is strong $\left(P_{\mathrm{CR}}^{\mathrm{Num}} / P_{\mathrm{CR}}^{\mathrm{Lin}} \approx 10\right.$ over $10 \mathrm{pc}$ from the center of the source). In the DiM the self-confinement effect is stronger than in the WNM and the CNM. Moreover it lasts over longer times, more than $50 \times t_{\frac{1}{2}}$.

We perform simulations for $10,100 \mathrm{GeV}$ and $10 \mathrm{TeV}$ CRs. At low energy the confinement effect is weak in both phases. At high energy $(10 \mathrm{TeV})$, we do not have solutions int the WNM. But the non-linear effect is very strong in the CNM and the DiM. The diffusion coefficient drops by a factor $10^{4}$ from the background one, the ratio $P_{\mathrm{CR}}^{\mathrm{Num}} / P_{\mathrm{CR}}^{\mathrm{Lin}} \approx 10$ over about $100 \mathrm{pc}$. The effect is still present after $10^{3} \times t_{\frac{1}{2}}$. See Brahimi et al. (2019) submitted.
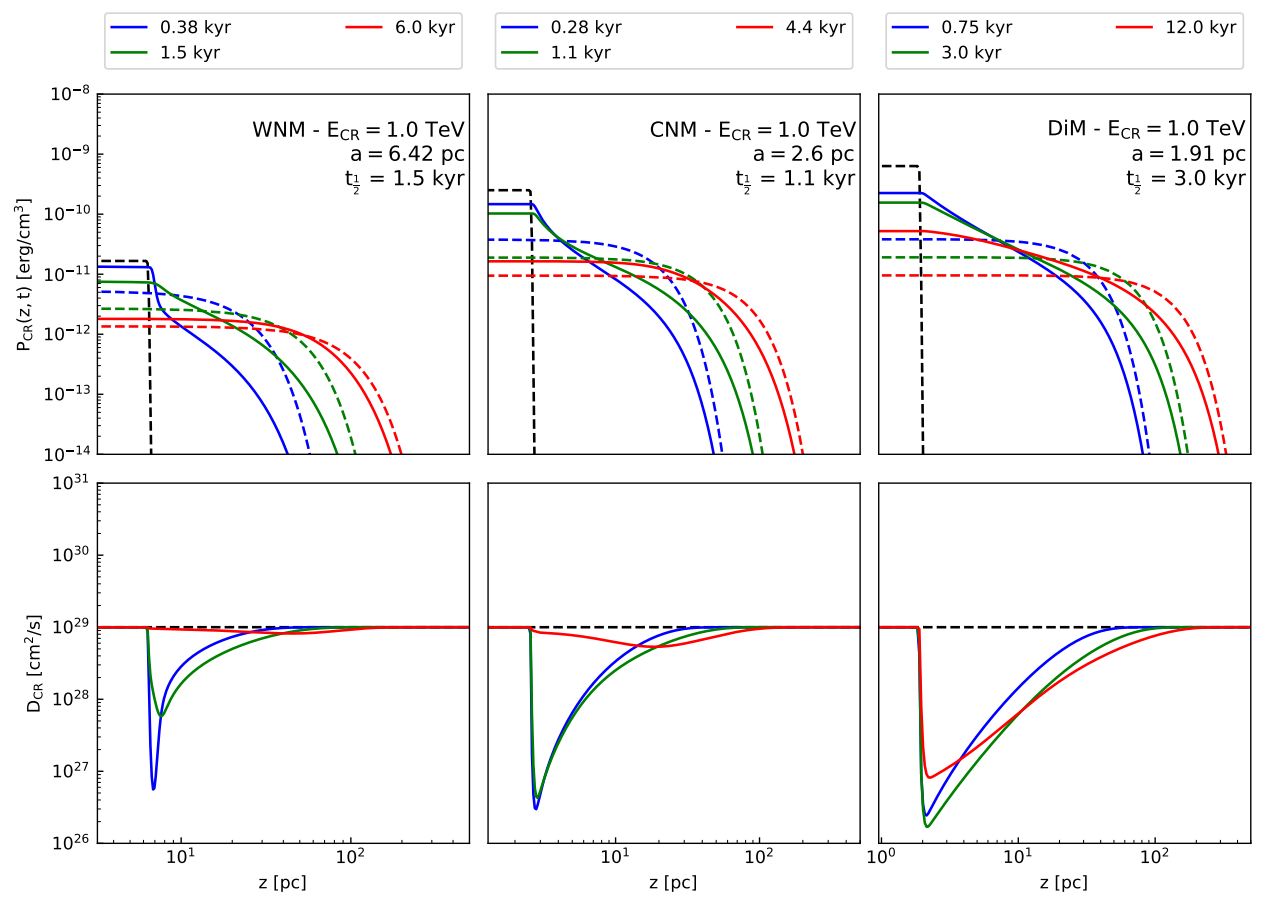

Figure 2: Numerical solutions of the CRs leakage problem. Upper sub-plots refer to CRs pressure $P_{\mathrm{CR}}$ evolution solutions in the WNM (left), CNM (middle), DiM (right). Black dashed line represent the initial pressure $\left(P_{\mathrm{CR}}^{0}\right)$. Colored solid lines (dashed lines) represent the numerical (test particle) solutions at $t_{\frac{1}{2}} / 4$ (blue), $t_{\frac{1}{2}}$ (green) and $4 \times t_{\frac{1}{2}}$ (red) respectively. Lower sub-plots refer to CRs diffusion coefficient $D$ solutions. Black dashed lines corresponds to the background diffusion coefficient. Colored solid lines corresponds to the modified diffusion coefficient following the color code used for pressure solutions

\section{Cosmic Rays grammage}

The CRs confinement around the accelerator can be well represented by the grammage $X$ 
linked with their residence time $\tau_{\text {res }}$ by the relation $X \approx 1.4 m_{p} n_{T} c \tau_{\text {res }}$. We calculated the CRs residence time from an average square of the diffusion distance

$$
z_{*}^{2}=\frac{\int_{0}^{\infty} z^{2} P_{\mathrm{CR}}\left(E, z, \tau_{\mathrm{res}}\right) \mathrm{d} z}{\int_{0}^{\infty} P_{\mathrm{CR}}\left(E, z, \tau_{\mathrm{res}}\right) \mathrm{d} z} .
$$

The results of the CRs grammage are presented in the figure (3) for the WNM, CNM and the DiM. In the WNM, we observe that the grammage decreases as $E^{-0.5}$ from $10 \mathrm{GeV}$ to $10 \mathrm{TeV}$ following the observationally inferred value meaning that background turbulence controls it. At $50 \mathrm{pc}$ from the center of the source, it is about $0.03 \mathrm{~g} / \mathrm{cm}^{2}$ for $10 \mathrm{GeV} \mathrm{CRs}$ and $0.003 \mathrm{~g} / \mathrm{cm}^{2}$ at $1 \mathrm{TeV}$. But we observe a different trend in CNM and DiM. In the CNM the grammage decreases as $E^{-0.5}$ from $10 \mathrm{GeV}$ to $1 \mathrm{TeV}$ but starts to increase above $1 \mathrm{TeV}$. At $50 \mathrm{pc}$ from the center of the source, the grammage is about $2 \mathrm{~g} / \mathrm{cm}^{2}$ at $10 \mathrm{GeV}$, decreases to $0.3 \mathrm{~g} / \mathrm{cm}^{2}$ and increases up to $10 \mathrm{~g} / \mathrm{cm}^{2}$ above $10 \mathrm{TeV}$. In the DiM, the effect of the grammage modification is even more important, the slope start to soften it from $100 \mathrm{GeV}$, reaches a minimum around $3 \mathrm{TeV}$ and increases above. At $50 \mathrm{pc}$ from the source the CRs grammage is about $20 \mathrm{~g} / \mathrm{cm}^{2}$ at $10 \mathrm{GeV}$, has a minimum of about $0.5 \mathrm{~g} / \mathrm{cm}^{2}$ around $3 \mathrm{TeV}$ and goes up to $20 \mathrm{~g} / \mathrm{cm}^{2}$ above $10 \mathrm{TeV}$.

These values are the signature of a significant CRs confinement effect around SNRs in the cold neutrals and molecular medium environnements.

\section{Multiphase simulations}

In order to probe more realistic configurations, we implemented a non-homogeneous medium setup (see Brahimi et al. 2019 submitted). We perform two simple multiphase setups. In the first case (setup : WNM-CNM), CRs are ejected by a SNR in a WNM phase and reach a CNM phase at $50 \mathrm{pc}$ from the center of the source. At $110 \mathrm{pc}$, they leave from the CNM phase and go again in the WNM. In the second case (setup : WNM-CNM-DiM), we put a DiM phase at the center of the CNM phase. We simulate the escape of $10 \mathrm{GeV}$ CRs, in both setups we observe a behaviour change in the transport properties of CRs.

In the WNM-CNM setup, we observe that at early times (10 kyrs) CRs seem to be absorbed by the CNM. Inside the cloud, the CR pressure is about $10 \%$ larger than the one in the case of a propagation in an homogeneous WNM. Slightly before the cloud, we observe a slight drop of the CR pressure. This behaviour can be explained by the modification of the CRs diffusion coefficient at the transition WNM-CNM which is 1.4 higher than in the homogeneous case. CRs diffuse faster in the CNM. At late times (170 kyrs), the CRs pressure in the first part of the simulation box ( $100 \mathrm{pc})$ is lower than in the WNM case $\left(P_{\mathrm{CR}}^{\mathrm{WNM}-\mathrm{CNM}} / P_{\mathrm{CR}}^{W N M} \sim 0.88\right)$ and start to increase after. To resume, the effect of the presence of a CNM cloud is to accelerate the diffusion process.

In the WNM-CNM-DiM setup, we obtain almost the same results at first order. Between the CNM and the DiM we observe the same changes in the CR transport properties but at an order of magnitude less. The presence of a DiM phase seems negligible when we have a WNM-CNM transition.

\section{Perspectives}

We are improving our code in the following directions : 


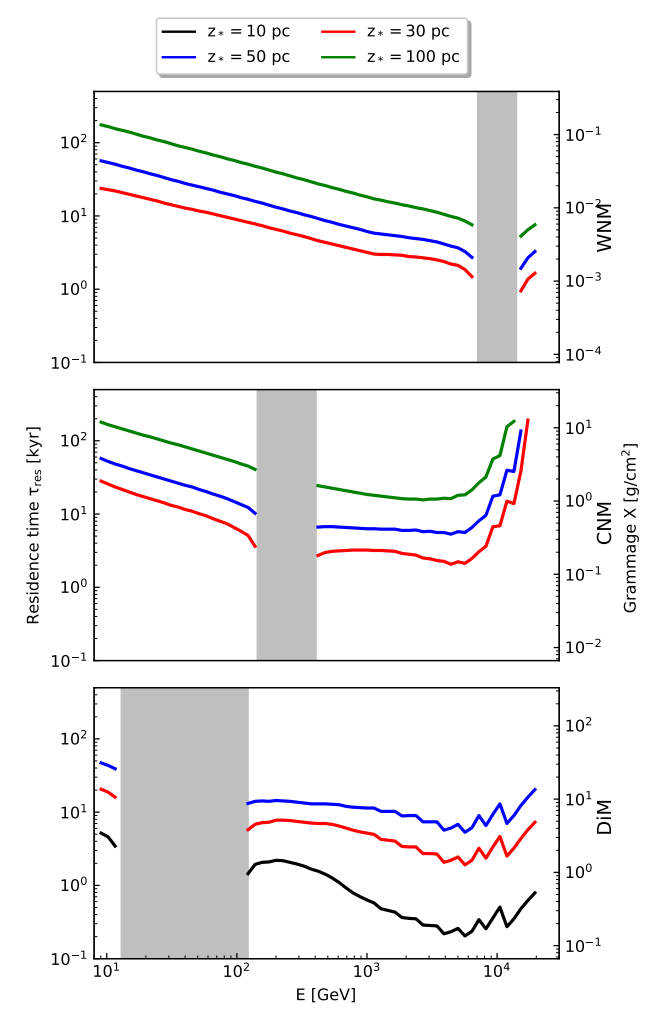

Figure 3: This figure shows the grammage (right vertical axis) and the residence time (left vertical axis) of CRs as function of their energy in three different phases of the ISM : WNM (top), CNM (middle) and DiM (bottom). Solid lines show the results obtained at different distances $z_{*}$ from the center of the source: green $(100 \mathrm{pc})$, blue $(50 \mathrm{pc})$, red (30 pc) and black (10 pc). The shaded region marks the no-wave propagation zone. Oscillations at high energy in the CNM and DiM are due to limited energy resolution used to reconstruct the different curves.

- Adding an adiabatic losses term in order to take in account the energy exchanges between the different populations of CRs. This term allows us to describe spectral evolution of CRs. $\gamma$-ray spectrum will be derived.

- Low energy CRs can lose energy by interacting with dense clouds via coulomb interactions. We are implementing a coulomb losses term in the CRs pressure equation which will also calculate the ionization rate (in $\mathrm{s}^{-1}$ ) due to the presence of CRs.

- CRs pressure gradient have important effects on the generation of Alfvén perturbations. We only considered foward waves. But in a multiphase case, it is possible to have space negative pressure gradients. We are consequently implementing a backward waves term.

- In the case of strong CRs pressure gradients, the dominant CRs can diffuse perpendicularly to the mean magnetic field lines. This imply to implement a second spatial dimension in our 
simulation.

- Other type of instabilities can be implemeted, especially the non-resonant streaming instability.

\section{Conclusion}

We show that CRs accelerated at SNRs shocks can have important impact over the plasma turbulence properties in the SNRs environnement. In the WNM we retrieve the observationnaly inferred values through the trend of the CRs grammage $\left(X \propto E^{-0.5}\right)$. But in the CNM and the DiM, high energy CRs tend to be strongly confined. This confinement effect is both a consequence from our CRs injection model strongly depending on the ISM properties and the energy of the CRC, and from the interactions between the self-generated magnetic perturbations with the ISM. At high energy ( $>100 \mathrm{GeV}$ ), the grammage obtained in the CNM and the DiM clearly shows that CRs can noticeably affect their environnement via turbulence generation.

\section{References}

[1] L. Nava, S. Gabici, A. Marcowith, G. Morlino, V.S. Ptuskin, Non-linear diffusion of cosmic rays escaping from supernova remnants - I. The effect of neutrals, MNRAS, [1606.06902]

[2] M.A. Malkov, P.H. Diamond, R.Z. Sagdeev, F.A. Aharonian, I.V. Moskalenko, Analytic solution for self-regulated collective escape of cosmic rays from their acceleration sites, APJ, [1207.4728]

[3] A. Lazarian, Damping of Alfvén waves by turbulence and its consequences: from cosmic ray streaming to launching winds, APJ, [1607.02042]

[4] S. Xu, H. Yan, A. Lazarian, Damping of magnetohydrodynamic turbulence in partially ionized plasma: implications for cosmic ray propagation, APJ, [1506.05585]

[5] R. Soler, M. Carbonell, J.L. Ballester, J. Terradas, Alfvén waves in a partially ionized two-fluid plasma, APJ, [1303.4297]

[6] A. Lazarian, J. Cho, Basic properties of compressible MHD turbulence: implications for molecular clouds, ASS, [0311372]

[7] P. Jean, W. Gillard, A. Marcowith, K. Ferriére, Positron transport in the interstellar medium, A\&A, [0909.4022]

[8] D. F. Cioffi, C. F. McKee, E. Bertshinger, Dynamics of radiative supernova remnants, APJ

[9] J. K. Truelove, C. F. McKee, Evolution of non-radiative supernova remnants, APJ

[10] J. Skilling, Cosmic ray streaming, MNRAS 